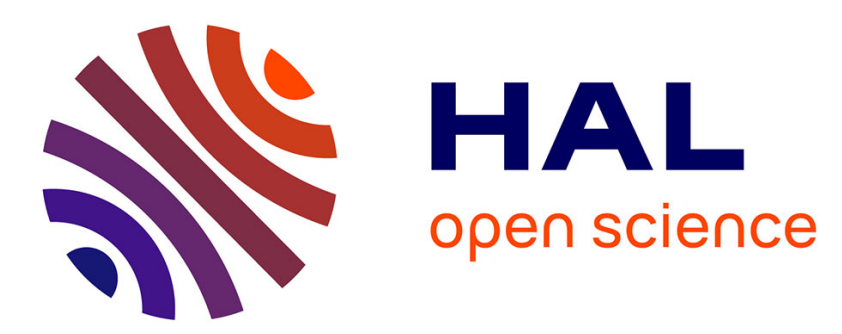

\title{
Vers un changement de paradigme économique dans le contexte post-pandémie?
}

Pierre Penet, Paul Jorion, Frédéric Lebaron

\section{To cite this version:}

Pierre Penet, Paul Jorion, Frédéric Lebaron. Vers un changement de paradigme économique dans le contexte post-pandémie?. Savoir / Agir , 2021, N 56, pp.63-70. 10.3917/sava.056.0065 . hal03472084

\section{HAL Id: hal-03472084 \\ https://hal.science/hal-03472084}

Submitted on 9 Dec 2021

HAL is a multi-disciplinary open access archive for the deposit and dissemination of scientific research documents, whether they are published or not. The documents may come from teaching and research institutions in France or abroad, or from public or private research centers.
L'archive ouverte pluridisciplinaire HAL, est destinée au dépôt et à la diffusion de documents scientifiques de niveau recherche, publiés ou non, émanant des établissements d'enseignement et de recherche français ou étrangers, des laboratoires publics ou privés. 
Grand entretien avec Paul Jorion

Propos recueillis par Pierre Pénet et Frédéric Lebaron

Vers un changement de paradigme économique dans le contexte post-pandémie?

Depuis des mois, les bourses battent des records mais la multiplication de faillites
d'entreprises est aussi le signe que des problèmes plus profonds existent. Quelle évaluation
faites-vous de l'état de l'économie et de la finance depuis le début de la pandémie?

Certains secteurs de l'économie ont évidemment été très profondément touchés par la pandémie, comme le commerce de détail et le secteur de la restauration et de l'hôtellerie. Quand on examine les données économiques, on voit ces derniers mois des rebonds dans certaines activités qui remontent jusqu'à $60 \%$ ou $75 \%$ de leur niveau d'avant-crise, mais sans toutefois atteindre les $100 \%$. Certaines pertes pourraient donc être irréversibles, et ce malgré les fonds publics investis pour relancer l'activité. S'agissant des marchés financiers, des effets en sens opposés se contrecarrent, augmentant les tensions, mais dans l'opacité. Dans le contexte actuel où les entreprises excluent la revalorisation des salaires, elles utilisent leurs liquidités en surplus pour racheter leurs propres actions et distribuer des dividendes aux montants obscènes, accélérant la concentration des richesses, alimentant du coup la spéculation par ceux qui ne savent que faire de leur richesse en excès.

Un décalage existe entre la capitalisation boursière des entreprises (mesurée par la somme totale du montant de leurs actions en circulation) et leur valeur dite «fondamentale », calculée sur la base de leur capacité à générer de la valeur ajoutée. Mais il est aujourd'hui malaisé de mesurer avec exactitude l'amplitude de la décorrélation des cours boursiers par rapport à l'économie réelle car les taux d'intérêt négatifs invalident le calcul classique de la valeur d'une action en tant que somme des dividendes « escomptés » (somme de leur valeur actualisée sur la base des taux d'intérêt du marché), déboussolant le baromètre classique de détection des bulles financières.

Cette opacité fait toute la différence avec le contexte qui précédait la crise des subprimes. Dans la période 2005-2008, le traçage des incertitudes financières était beaucoup plus clair, notamment concernant la surchauffe du marché immobilier. Certains comme Wynne Godley, Nouriel Roubini, Steve Keen et moi-même ont perçu des relations claires de cause à effet et ont cherché à alerter les autorités sur le danger de catastrophe financière ${ }^{1}$. Ces risques étaient bien perceptibles même si la grille d'interprétation des faits que procurait la « science » économique tendait à les rendre invisibles ; on pense en particulier à la croyance au sein de l'équipe d'Alan Greenspan à la Réserve fédérale que ce que certains interprétaient comme une bulle immobilière aux États-Unis était en fait un mirage causé par le moindre impact de l'informatisation sur la construction neuve que sur l'ensemble des autres secteurs de l'économie. Le contexte actuel est bien différent car les incertitudes ne sont pas cantonnées à un secteur bien délimité de la finance, comme c'était le cas pour les titres adossés à des prêts au logement subprimes, mais sont diffuses dans la totalité de l'économie et de la finance, ce qui conduit à poser un diagnostic de crise mais sans déboucher pour autant sur un pronostic définissable précisément. D'autant que la fragilité dans notre contexte pandémique n'est pas que financière mais aussi politique et sociale, comme on le voit en France avec la popularité

\footnotetext{
${ }^{1}$ Paul Jorion, 2007, Vers la crise du capitalisme américain ?, Paris, La Découverte, 2007.
} 
croissante des thématiques identitaires dans le débat public et les conséquences que cela pourrait avoir à l'occasion des prochaines échéances électorales, dans l'élection d'une candidate au programme proto-fasciste, au sens propre du terme.

Que penser des scandales financiers récents comme la controverse GameStop ou la faillite du fond spéculatif Archegos?

Ces incidents ne relèvent pas de l'extraordinaire. Les pertes (700 millions pour UBS avec l'affaire Archegos) sont de l'ordre du business as usual. L'agitation autour de GameStop renvoie quant à elle à des stratégies marginales mais classiques dites de pump and dump visant à pousser le cours d'une action loin de son fondamental qui, étant jugé comme sa valeur « raisonnable », agit comme un attracteur pour son cours. Dans un cas comme dans l'autre, nous sommes en présence de phénomènes vieux comme les bourses elles-mêmes. Le fait que les protagonistes de la controverse Gamestop soient des particuliers, généralement novices en matière de finance, a conduit certains commentateurs étrangers au monde de la finance, à lire une analogie entre la farce Gamestop et le mouvement Occupy Wall Street. Le fait que le courtier qui a permis à des novices de passer gratuitement leurs ordres sur Gamestop s'appelle Robinhood (Robin des Bois) ${ }^{2}$, a pu faire croire aux croquants que quelque chose se passait qui s'apparenterait à une irruption salutaire de la justice sociale sur les marchés boursiers. Si le retour de la justice sociale est en effet essentiel, la Bourse n'est en tout cas pas le lieu privilégié où l'on peut espérer la voir advenir !

Vu la familiarité des marchés avec l'irruption occasionnelle de croquants dans le jeu boursier, celle-ci sera sans effet sur la finance. L'ébullition Gamestop reste en soi intéressante en tant que trait de notre époque marquée par la multiplication de jacqueries et de mobilisations d'inspiration populiste. Ces mobilisations dirigées contre les représentants politiques (le mouvement des Gilets jaunes est dans toutes les mémoires) visent aussi les acteurs financiers et les experts économiques. L'affaire GameStop, au même titre que l'irruption de la Théorie Moderne de la Monnaie, signale l'envie d'en découdre sur tous les fronts d'une partie significative de la population, sa familiarité plus ou moins grande avec les mécanismes financiers apparaissant en l'occurrence comme une variable indifférente.

\section{Quelle interprétation donner à l'engouement actuel autour de la Théorie Moderne de la Monnaie ? Comment situez-vous ces travaux, notamment ceux de Stéphanie Kelton, dans le paysage des théories économiques hétérodoxes?}

On parle beaucoup aujourd'hui de la Théorie Moderne de la Monnaie (TMM), notamment aux États-Unis où certaines personnalités de la gauche critique comme Alexandria Ocasio Cortez en ont fait la promotion. Plusieurs livres emblématiques ont récemment été traduits en français, notamment celui de Stéphanie Kelton qui apparaît comme une cheffe de file de ce courant $^{3}$. Malheureusement, à y regarder de plus près, ces travaux ne sont pas organisés autour d'une théorie économique cohérente mais apparaissent plutôt comme un assemblage hétéroclite de propositions économiques hétérodoxes empruntées à des auteurs de différentes époques, du dix-neuvième au vingt et unième siècle, aux vues souvent inconciliables, comme Georg Friedrich Knapp (1842-1926), le John Maynard Keynes (1884-1946) de A Treatise on

\footnotetext{
${ }^{2}$ La plateforme de courtage Robinhood n'est gratuite que parce que qu'elle vend l'information que constituent ces ordres à des opérateurs susceptibles d'en tirer parti.

${ }^{3}$ Stephanie Kelton, Le mythe du déficit, Paris, Les liens qui libèrent, 2021 ; Pavlina R. Tcherneva, La garantie d'emploi. L'arme sociale du Green New Deal. Paris, La Découverte, 2021.
} 
Money (1930), où il défendait un point de vue sur la monnaie qu'il s'empressa d'abandonner aussitôt, Nicholas Kaldor (1906-1986) ou Wynne Godley (1926-2010). Or, aucune des propositions de ce courant ne tient la route.

La proposition phare de la TMM est qu'un État peut créer autant de monnaie qu'il le souhaite, sans danger particulier pour son système financier ou son économie. Le seul danger de la création monétaire résiderait dans l'inflation. C'est pourtant oublier qu'un État créant de la monnaie au-delà de ce qui est perçu par l'ensemble des autres nations comme la richesse effective de son économie devra relever le taux de ses émissions obligataires, les investisseurs incluant une prime de risque toujours plus élevée dans les taux qu'ils exigent, d'où un coût pouvant devenir exorbitant pour l'État et barrant l'accès au crédit pour l'économie locale. La seule monnaie au monde pour laquelle la TMM semble valide sous ce rapport, est le dollar américain en raison de son statut de devise de référence. Mais la création illimitée de dollars se ferait aux dépens de l'ensemble des autres monnaies, une politique qui apparaît donc risquée car elle ne manquerait pas de liguer les autres nations contre les États-Unis.

Une autre thèse centrale de la TMM est l'affirmation que l'État peut atteindre le plein-emploi en créant de la monnaie, le montant exact étant à déterminer empiriquement, une affirmation imputée à Keynes par malentendu. Cette thèse a perdu toute vraisemblance aujourd'hui pour des raisons non-monétaires, à savoir du fait de la numérisation et de la robotisation du travail qui a fait de la perspective d'un retour au plein emploi dans les économies développées, un simple mirage.

La TMM adopte aussi la thèse « chartaliste » contestable qui voit l'origine de l'argent non dans le troc mais dans la dette et dans le système d'imposition mis en place par les États. Les anthropologues ont pourtant eu l'occasion de documenter la présence de biens utilisés comme « moyen générique de troc » dans des dizaines de sociétés traditionnelles non-étatiques, dont il est raisonnable de supposer qu'elles ont précédé historiquement l'apparition des États. On pense pour un excellent exemple à la monnaie de sel des Baruya de Nouvelle-Guinée, dont Maurice Godelier a rendu compte de manière exemplaire ${ }^{4}$.

Le seul représentant de la TMM dont je me sente proche est Hyman Minsky, dont j'ai utilisé le modèle du cycle du crédit dans mon compte-rendu de l'évolution de la crise des subprimes ${ }^{5}$, lequel est en général considéré comme « post-keynésien », mais à tort, sa pensée étant strictement keynésienne, comme ce fut le cas aussi pour Joan Robinson et Piero Sraffa, les rédacteurs de facto de certains chapitres de la Théorie générale de l'emploi, de l'intérêt et de la monnaie (1936). Geoff Tily a raison quand il affirme que la pensée de la plupart des supposés « post-keynésiens » est sans rapport aucun avec l'œuvre de Keynes ${ }^{6}$. Cela vaut également pour les tenants de la TMM.

Pour revenir à l'analogie proposée plus haut, le développement de la TMM est, comme pour l'affaire GameStop, l'irruption de non-spécialistes convaincus de renouveler la compréhension de la finance, mais au prix d'une simplification abusive de l'interprétation des

\footnotetext{
${ }^{4}$ Maurice Godelier, «La “'monnaie de sel” des Baruya de Nouvelle-Guinée », L'Homme, vol. 9, n 2, 1969, p. $5-37$.

${ }^{5}$ Paul Jorion, L'implosion. La finance contre l'économie : ce que révèle et annonce la « crise des subprimes », Paris, Fayard, 2008, p. 28-34

${ }^{6}$ Geoff Tily, Keynes Betrayed: The General Theory, the Rate of Interest and «Keynesian » Economics, London, Palgrave Macmillan, 2007.
} 
mécanismes financiers. L'affaire GameStop et la TMM remettent en mémoire le «système de Brown ». John Brown était un médecin britannique du dix-huitième siècle qui prétendait simplifier l'évaluation du niveau de santé des individus au travers d'un critère unique d' « excitabilité ». Brown avait ainsi conçu une jauge allant de 0 à 80 . Toute déviation par rapport à 40, la mesure du niveau de santé idéal, se traduisait par l'administration d'un médicament : un sédatif pour une excitabilité trop grande ou un stimulant pour une excitabilité trop faible. Cette méthode a dans un premier temps suscité un grand intérêt chez les étudiants en médecine qui y voyaient le moyen idéal de raccourcir les études médicales. Le souvenir du système de Brown s'est bien entendu rapidement estompé. La TMM procède d'une même sorte de populisme : les supposés marionnettistes du système dissimuleraient soigneusement aux yeux du peuple les ficelles qui leur permettent de les faire danser. La réalité est que ni les uns, ni les autres, ne comprennent le véritable fonctionnement des choses : le déficit dans la connaissance est largement partagé.

Ce «populisme de la connaissance » ne vient-il pas aussi en réaction à certains dogmes financiers malmenés durant la pandémie? Plutôt que d'y voir un processus venant du bas, ne faut-il pas y voir surtout la marque d'une crise affectant les croyances économiques des autorités financières et monétaires?

Ces jacqueries semblent en effet liées à une sorte d'épuisement des cadres de compréhension de l'économie et de la finance. Le fait que les théories économiques fausses soient martelées sans discontinuer souligne que l'ensemble des savoirs économiques sont à réévaluer. La « science » économique est à vue de nez à $60 \%$ correcte et à $40 \%$ fausse. L'incompréhension est totale, par exemple, du mécanisme de la formation des taux d'intérêt ${ }^{7}$.

Contrairement à ce que suggère leur dénomination de «non conventionnelles », les politiques monétaires qualifiées ainsi sont au banquier ce que sont le marteau au charpentier et le tournevis à l'électricien. Le procédé consistant pour un État à injecter de la monnaie ou à en retirer par l'émission d'obligations ou par rachat sur le marché secondaire d'obligations pour influer sur le niveau des taux à moyen et à long terme sont des stratégies tout à fait classiques de l'histoire monétaire. Pourtant, du fait d'une occultation de la réalité sociale de nos sociétés, plutôt que de stimuler l'économie réelle, ces politiques ont surtout servi à gonfler le portefeuille des spéculateurs. Ce décalage entre les effets attendus et les effets réels provient de la croyance naïve qu' on retrouve chez les libéraux depuis Jean-Baptiste Say selon laquelle l'offre créée la demande par un effet de trickle down (en français : ruissellement).

Une vérité de base est désormais ignorée : que la croissance consiste en une hausse du PIB, que le PIB est la somme des valeurs ajoutées, que la valeur ajoutée est ce que l'on appelle aussi le profit et que seuls les industriels et les marchands font du profit. Et que si l'on veut dès lors trouver le moyen que la croissance se manifeste en achat de produits à partir d'un pouvoir d'achat dans la population, il faut que les industriels et les marchands acceptent de se départir d'une partie au moins de leurs profits sous la forme de salaires accordés à des travailleurs.

Il y a une naïveté dans le fait de croire que nous vivons dans une société idéale où les profits faits par les industriels et par les marchands vont automatiquement se redistribuer de manière

\footnotetext{
${ }^{7}$ Voir Paul Jorion, «Un mécanisme de stabilité punitif », Le Monde, 7 mars 2011.
} 
effective en augmentation de salaires, et donc en gains pour l'économie par le biais du pouvoir d'achat.

Les choses se sont encore aggravées dans les années 1970 quand une invention du cabinet McKinsey \& Co a permis aux industriels de conclure une alliance avec les détenteurs de capitaux : les capitalistes. Jusque-là, les industriels et les capitalistes (que Marx confondait à tort), avaient des intérêts divergents.

Un bref rappel en ces matières n'est jamais superflu. Si on revient aux origines du monde industriel, il y a toujours eu trois parties en présence : d'un côté les industriels ou dirigeants d'entreprises ; d'un autre les capitalistes qui avancent le capital et récoltent les rentes que sont intérêts et dividendes; enfin les travailleurs percevant un salaire en échange de leur labeur. Jusque dans les années 1970, les travailleurs pouvaient opérer des alliances ponctuelles avec les dirigeants d'entreprises, soit éventuellement même avec les capitalistes, afin de défendre leurs intérêts. Cette logique tripartite a disparu lorsque le bureau d'études McKinsey inventa en 1975 la stock-option qui aligne les intérêts des industriels et des capitalistes. Les stockoptions seront ainsi distribuées aux industriels et aux dirigeants d'entreprises afin de les faire bénéficier eux aussi de la hausse des cours boursiers. À partir de là, ces derniers vont privilégier les gains de court terme en bourse par rapport à l'avenir à long terme de leur entreprise. Ces inflexions vont générer des catastrophes bien connues à partir de la bulle des startups et de l'internet. La faillite de la compagnie Enron, exemplaire aussi en tant que lightasset ou lean corporation, une firme aux infrastructures minimales, un autre concept McKinsey, reste aujourd'hui un exemple classique de ce capitalisme à la rapacité accrue.

Pour éviter que les politiques d'assouplissement quantitatif ne gonflent simplement les bulles spéculatives, un mécanisme doit garantir que ces injections de liquidités se traduisent par des augmentations de salaires plutôt que par la distribution de dividendes. Tout cela n'est pas révolutionnaire : c'est précisément le sens du discours tenu par Joe Biden, au Congrès américain :

«La pandémie n'a fait qu'aggraver les choses. 20 millions d'Américains des classes moyennes et populaires ont perdu leur travail durant la pandémie pendant que 650 milliardaires voyaient leurs revenus croître de plus de mille millards de dollars (...). Mes chers concitoyens, la théorie du ruissellement n'a jamais marché. Il est temps de stimuler l'économie par le bas et par le milieu. » (The White House, « Remarks by President Biden in Address to a Joint Session of Congress », Speeches and Remarks, 29 avril 2021) ${ }^{8}$

Cette déclaration de la part d'un président américain représente un véritable basculement. Il n'y avait qu'à observer la mine réjouie et les sourires approbateurs des représentants de l'aile gauche du Parti démocrate comme Bernie Sanders et Alexandria Ocasio-Cortez.

\section{Comment interprétez-vous la séquence politique actuelle aux États-Unis ? Des plans de relance massifs ont été annoncés, suscitant l'inquiétude chez des économistes centristes}

\footnotetext{
${ }^{8}$ «The pandemic has only made things worse. Twenty million Americans lost their job in the pandemic, working- and middle-class Americans. At the same time, roughly 650 billionaires in America saw their net worth increase by more than $\$ 1$ trillion, in the same exact period. [...] My fellow Americans [...] trickle-down economics has never worked. It's time to grow the economy from the bottom and the middle out ».
} 


\section{comme Olivier Blanchard mais aussi l'enthousiasme chez des économistes de gauche comme Paul Krugman. Est-on véritablement au seuil d'un nouveau New Deal?}

J'y vois la marque d'une recomposition politique importante après les quatre années de présidence Trump. On peut voir dans certains sondages qu'une partie de la base Républicaine est en train de changer de bord sur les questions économiques ${ }^{9}$. Cette fraction de l'électorat est très importante car elle regroupe des électeurs qui avaient voté Obama mais qui s'étaient ralliés à Trump par la suite. Ce sont des électeurs qui ont attendu, en vain, qu'Obama soit le nouveau Roosevelt. Il est remarquable que Biden ait déjà réalisé en 100 jours plus de mesures de gauche que Obama en huit ans. Obama a causé d'autant plus de déception que fin 2008, juste avant l'élection d'Obama, les Républicains semblaient prêts à envisager des mesures économiques courageuses. Les Républicains étaient absolument affolés par la crise des subprimes et percevaient la nécessité d'opérer un changement de cap économique vers la gauche. Mais Obama a pratiqué une politique centriste, voire même de centre droit. Les politiques de l'administration Biden ne sont finalement pas tellement surprenantes car elles dénotent une volonté de ne pas répéter les erreurs la précédente administration démocrate. Cela s'est vu notamment dans des propositions de politiques économiques portées par Elizabeth Warren et Ocasio-Cortez auxquelles une majorité d'Américains, surtout parmi les plus jeunes, pouvaient s'identifier. C'est de cette façon qu'on peut comprendre comment Biden, auparavant identifié à un homme politique de centre droit, propose désormais un retour à l'État interventionniste.

L'évolution de Biden est aussi une façon de reconquérir l'électorat séduit par Trump, notamment ceux qu'on appelle de manière grossièrement insultante le trailer trash, le « détritus des roulottes », c'est-à-dire la population blanche paupérisée vivant dans des mobile homes. Le plus remarquable dans le discours de Biden, c'est quand il s'adresse à la caméra et il qu'il déclare, en substance : « Vous les personnes sans diplôme, vous qui n'avez pas le bac, on va faire en sorte qu'il y ait des dizaines de millions de boulots pour vous ». Aucun président ne s'était adressé directement et de cette façon à cet électorat depuis Lyndon Johnson et sa « Great Society » en 1964.

\section{Par comparaison, comment jugez-vous du contexte européen qui semble marqué par un plus fort degré d'inertie?}

Il y a un basculement en Europe qui va dans la direction opposée. En France, notamment, où paradoxalement depuis l'épidémie, les problèmes économiques ont été relégués au deuxième ou au troisième rang, derrière les enjeux de sécurité et la question des rapports entre « communautés », dont la manifestation dans le monde universitaire est la polémique autour de l' «islamo-gauchisme ». Il faut souhaiter que l'évolution présente des États-Unis ne passe pas inaperçue en Europe et finisse par détourner l'attention des obsessions franco-françaises du moment. Mais il y a bien des raisons d'être pessimiste sur la capacité de la gauche européenne et tout particulièrement française à porter un discours ambitieux sur les questions économiques. La principale raison est évidemment que les partis de gauche restent très divisés sur l'économie. Les points de vue sont tellement différents qu'ils sont inconciliables, du moins à court ou moyen terme. François Ruffin a l'amabilité de me compter comme membre de son équipe et j'espère qu'une personnalité de son calibre finira par émerger.

\footnotetext{
${ }^{9}$ Chris Kahn, « Many Republican voters agree with Biden - "trickle-down economics" has failed », Reuters, 29 avril 2021.
} 
Mais la mise en place de politiques de gauche ne passera peut-être pas par des solutions classiques du type « union de la gauche » mais plutôt par des accidents du type Biden, c'est-àdire l'arrivée au pouvoir d'acteurs politiques centristes ou de centre droit qui seront conduits à gauchir leurs discours, par conviction ou par pur opportunisme, afin de contrer la montée du populisme d'extrême-droite. Il faut donc parier sur l'inattendu : de la même manière que la gauche au pouvoir a fait ces dernières années le contraire de la gauche, sans compter qu'elle a instauré avec Pierre Bérégovoy le néolibéralisme en France, on aura peut-être de manière inattendue un centre droit intelligent venant conforter l'État-providence, en inscrivant par exemple son existence dans la Constitution. Malheureusement, je ne crois pas que la France soit prête d'ici à 2022 à appliquer des politiques comparables à celles de l'administration Biden pour éviter le risque d'accession au pouvoir d'un régime proto-fasciste lors des prochaines élections présidentielles.

\section{Il y a quand même des spécificités institutionnelles propres à la zone euro. L'inertie européenne n'est-elle pas aussi liée aux contraintes européennes fortes en matière de finances publiques (les règles budgétaires de Maastricht) qui compliquent fortement la mise en ouvre de politiques économistes ambitieuses?}

L'euro est en effet une monnaie inachevée et bancale en l'absence de Trésor européen à l'échelle de l'ensemble de la zone monétaire, créant l'effet pervers de banques centrales nationales émettant de la dette à des taux de coupon différents. La zone euro tolère également en son sein l'existence de purs paradis fiscaux comme le Luxembourg ou de paradis fiscaux partiels comme l'Irlande, la Belgique ou les Pays-Bas, minant la politique fiscale de leurs voisins. Tout cela est source de fragilité comparativement aux États-Unis qui règlent ces problèmes d'intégration budgétaire par une remise à zéro annuelle des compteurs dans les relations entre les différents états de la fédération et qui, en raison du statut particulier du dollar comme monnaie de réserve internationale, peuvent sans risque financer leurs déficits par de la création monétaire. S'agissant des règles budgétaires européennes, elles ont fortement contraint les choix politiques ces dernières années. Mais si les États européens ont jusqu'à présent cherché à se conformer aux critères de Maastricht, rien n'indique que le statut quo perdure dans les mois et les années à venir. Les règles budgétaires ont été suspendues durant la pandémie et il suffira d'un accord entre le Président français et la Chancelière allemande pour entériner la disparition de ces dogmes dans les mois à venir. À mon sens, cette évolution aura lieu. La situation européenne reste donc plus ouverte qu'elle n'y paraît. La dimension déterminante dans l'après crise sanitaire sera la dimension politique. 